\title{
Analysis and Research on characteristics and corrosion resistance of graphene attached to copper surface
}

\author{
HE Rong-bu ${ }^{1,2, *}$ \\ ${ }^{1}$ Electric Power Research Institute of Guizhou Power Grid Co., Ltd, Guiyang, 550002; \\ ${ }^{2}$ Key Laboratory of Ice Disaster Prevention of Guizhou Power Grid Co., Ltd, Guiyang, 550002
}

\begin{abstract}
The adhesion of graphene on copper surface was studied, and its chemical characteristics and corrosion resistance were analyzed. The results of polarization curve analysis show that the corrosion resistance of graphene film is the best when the growth time is $7 \mathrm{~min}$. electrochemical impedance spectroscopy shows that the corrosion resistance of copper substrate is improved by 17 times. The graphite strips prepared at $7 \mathrm{~min}$ have the best uniformity and continuity. The graphite residue at $4 \mathrm{~min}$ is thin but discontinuous. The graphite strips at $10 \mathrm{~min}$ have the thickest thickness but the most grain boundary defects. The optimal process parameters were determined as follows: AR / CH4 / H2 flow rate ratio 600 / 40 / 30 $\mathrm{SCCM}$, temperature $1000^{\circ} \mathrm{C}$, reaction time $7 \mathrm{~min}$.
\end{abstract}

\section{Preface}

Graphene can be prepared on any metal surface by chemical vapor deposition (CVD). Copper is selected as the matrix, because copper matrix acts as a catalyst in the process of graphene formation, and the nucleation of graphene crystal nucleus is prior to the grain boundary or defect of copper matrix. In addition, there is a problem of lattice adaptation between graphene and copper grains. As introduced in the introduction, there are two main growth mechanisms of graphene in chemical vapor deposition, one is surface adsorption growth (represented by $\mathrm{Cu}$ ), the other is carburization and carbon evolution (represented by Ni). The growth mechanism of graphite strips on the copper surface is not shown in Fig. 1. The six membered ring structure of graphite thin grains nucleates at the grain boundary or defects on the copper surface, and then continuously connects and grows.

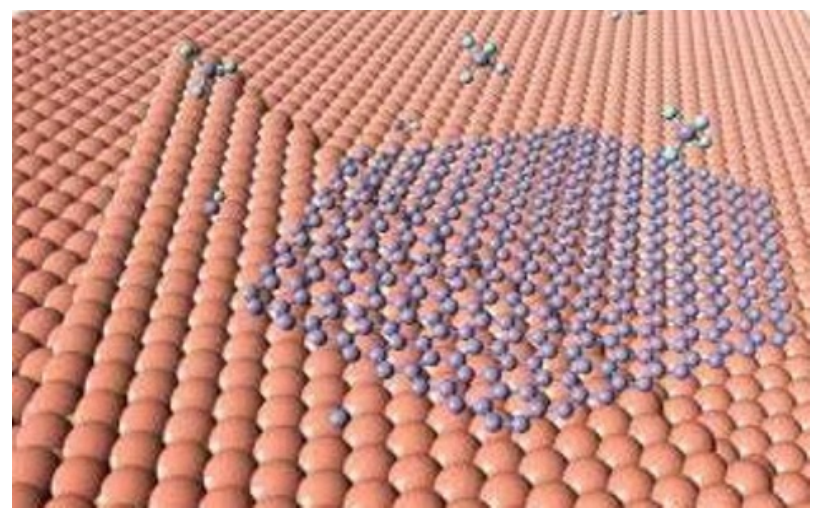

Fig 1 The growth mechanism of graphene on copper

The key to the corrosion resistance of graphene reinforced matrix is the preparation of graphene, that is, whether the graphene is uniform and continuous. The main factors affecting the growth of graphene are: the purity and surface finish of copper matrix, carrier gas $\mathrm{H} 2$, temperature and rate of temperature rise and fall, growth time, gas flow rate and gas ratio. In order to explore the electrochemical characteristics and corrosion resistance of graphene coated copper metal, the following tests and demonstration were carried out in this paper.

\section{FIGURES AND TABLES}

\section{1 electrochemical test}

The polarization curve and electrochemical impedance spectrum of five samples in Table 3-1 were tested by gamry reference 600 electrochemical test system. Three electrode systems were used, saturated mercury electrode was used as reference electrode, platinum net was the opposite electrode, electrolyte solution was 0.1 $\mathrm{mol} / \mathrm{l} \mathrm{NaCl}$ solution, and the electrode area was $1 \mathrm{~cm} \times 1$ $\mathrm{cm}$, and the experiment was carried out at room temperature.

Table 1 Five samples for electrochemical test .

\begin{tabular}{|c|c|}
\hline No & Sample \\
\hline 1 & pure $\mathrm{Cu}$ \\
\hline 2 & $\mathrm{Cu}$ after heat treatment \\
\hline 3 & 10 min graphene coated $\mathrm{Cu}$ \\
\hline 4 & 7 min graphene coated $\mathrm{Cu}$ \\
\hline 5 & 4 min graphene coated $\mathrm{Cu}$ \\
\hline
\end{tabular}

\footnotetext{
* Corresponding author: 554146767@qq.com
} 
The corrosion resistance of metal has a great relationship with its microstructure and structure. The main purpose of this study is to prepare graphene film on the surface of copper sheet to improve the corrosion resistance of copper. The preparation of graphene is carried out at high temperature. The heat treatment under this condition will change the microstructure of copper. In order to eliminate this factor, the corrosion resistance of copper is improved In this study, a group of control tests were added to remove the graphene film on the copper surface after vapor deposition with fine sandpaper. Moreover, due to the influence of many factors, the electrochemical test results are unstable. In order to enhance the repeatability of the experiment and make the test results more convincing, three parallel samples are set under each condition, and the average value is taken to make the final comparison.s.

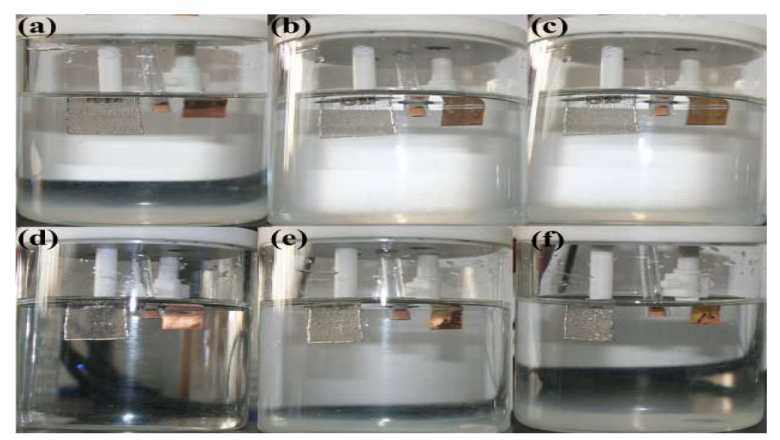

Fig 2 The diagram of electrochemical test process

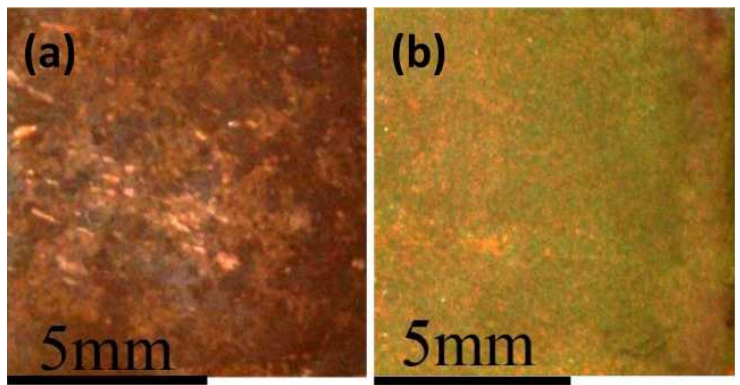

Fig3 The topography of the pure copper and graphene coated copper after test

After the electrochemical corrosion test, the samples of graphene coated copper and pure copper were dried, and their corrosion morphologies were compared. Fig. 2 is the process diagram of electrochemical corrosion test, in which Fig. (a) (b) (c) the process diagram of graphene coated copper In the experimental diagram of the sample at the beginning, middle and end of the polarization curve test, it can be seen that there is almost no change on the surface of the copper sheet, but the color is slightly deepened. Figure (d) (E) (f) is the experimental diagram of the pure copper sample at the beginning, middle and end of the polarization curve test, and it can be clearly seen that black substances fall off on the surface of the copper sheet in figure (E), In figure (f), the black substance sinks into the bottom of the cup and dissolves after a period of time.

\section{2 polarization curve}

The potentiodynamic polarization curves were measured at room temperature, and the scanning rate was $0.5 \mathrm{mv} / \mathrm{s}$. Among them, the anode current density is the parameter of copper dissolution rate, that is, the anodic oxidation reaction: cu-cu2 $++2 \mathrm{E}-$, and the cathode current density is the parameter of oxygen reduction reaction, that is, the cathode reduction reaction: $02+2 \mathrm{~h} 20+4 \mathrm{E}-$ $4 \mathrm{OH}$. The corresponding potential at the junction of cathode and anode area is the corrosion potential Ecorr of the sample, which reflects the corrosion sensitivity. The test results of five groups of parallel samples are shown in Figure 4, and the final comparison is shown in Figure 5.
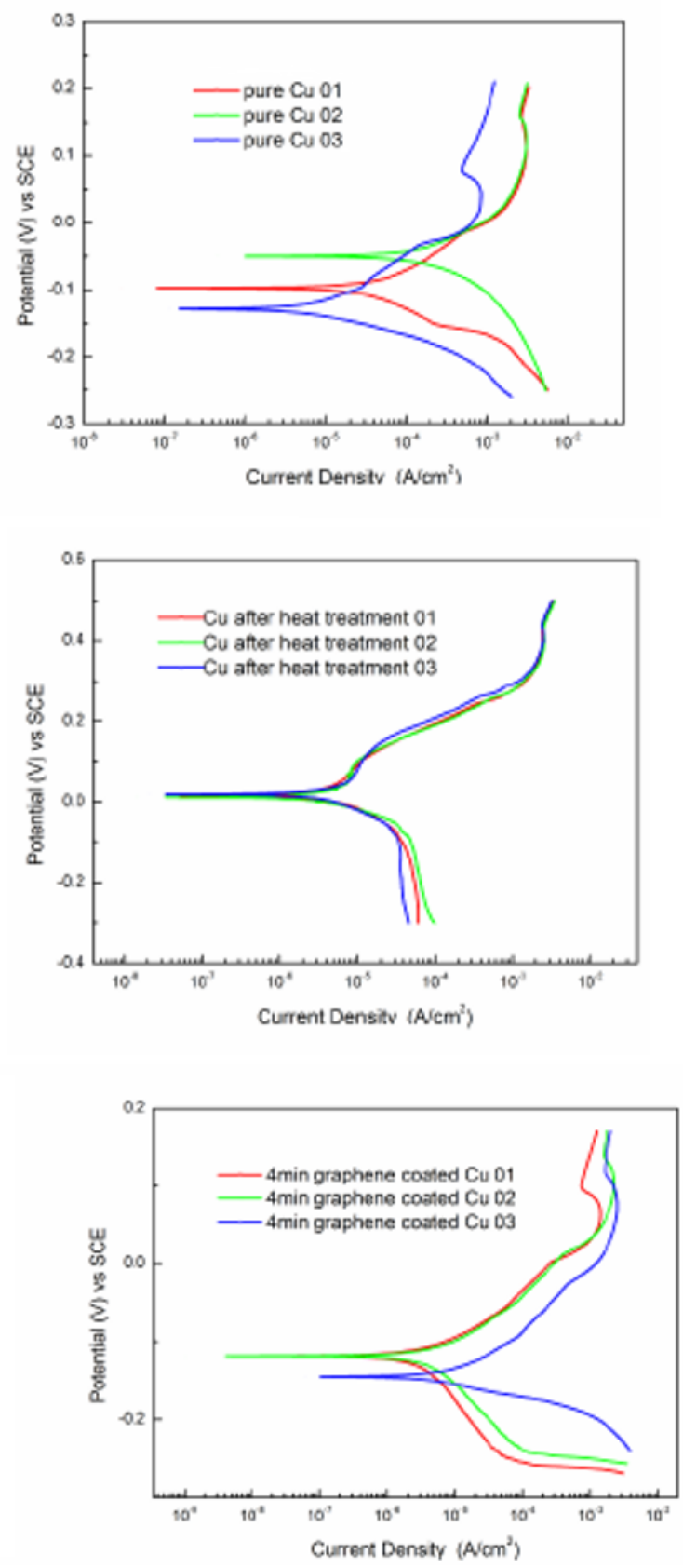

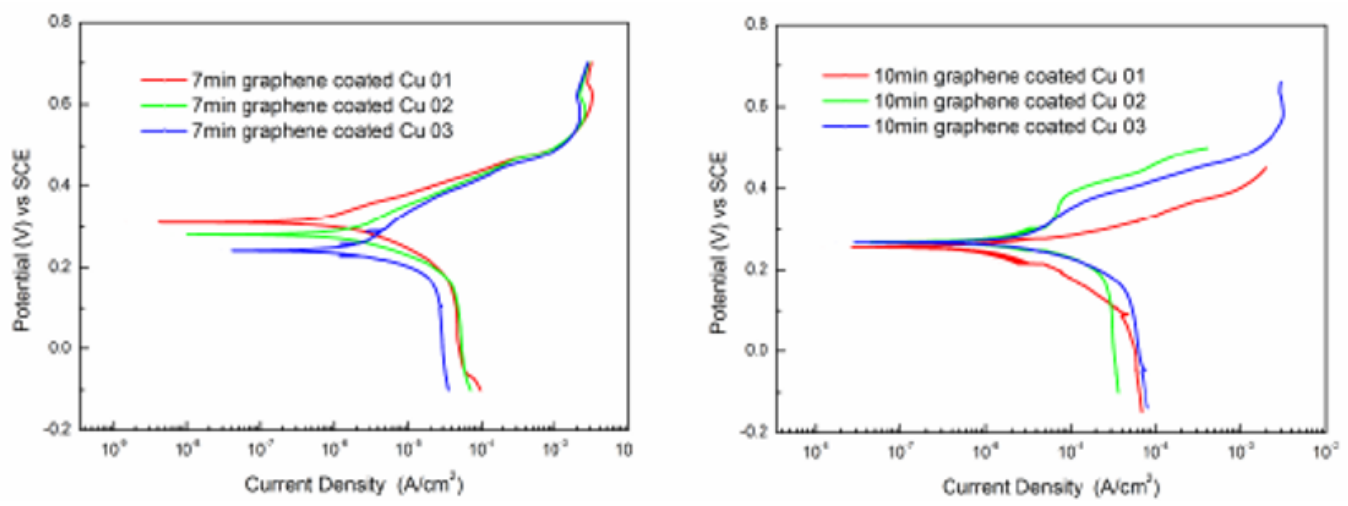

Fig 4 The polarization curve of five parallel groups

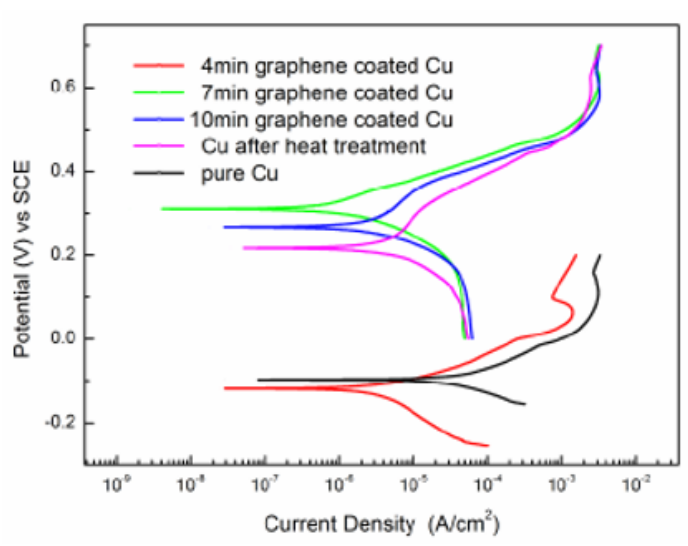

Fig 5 The final contrast chart of polarization curve

As shown in Fig. 4, the curves of each group of parallel samples do not fluctuate much, which indicates that the stability and repeatability of the test are good. Therefore, the intermediate value is taken to make the final comparison and figure 5 is obtained. It can be seen from Figure 5 that the corrosion current of samples with graphite dilute growth time of $4 \mathrm{~min}, 7 \mathrm{~min}$ and $10 \mathrm{~min}$ is lower than that of pure copper, and the average current of $7 \mathrm{~min}$ green curve is the lowest, which indicates that the sample surface has less conductive path, the least amount of charge, and is not easy to be corroded, and its Ecorr is the largest, greater than $0.3 \mathrm{~V}$, which indicates the corrosion resistance of the sample In other words, graphene film can effectively inhibit the corrosion of copper substrate. Compared with the original pure copper, the current density of the heat-treated pure copper sample also decreases significantly, and Ecorr increases by about $0.3 \mathrm{~V}$. The results show that the recrystallization process of copper sheet at high temperature makes the grain more uniform, reduces the structural defects and enhances the corrosion resistance significantly. However, the corrosion resistance of samples with $10 \mathrm{~min}$ and $7 \mathrm{~min}$ growth time of graphene is better than that of pure copper after heat treatment, which eliminates the influence of heat treatment factors. It shows that the graphene films with $10 \mathrm{~min}$ and $7 \mathrm{~min}$ growth can protect the substrate from corrosion.
However, the average current of the sample grown for 4 min is significantly lower than that of pure copper, but its Ecorr is lower than that of other samples, which indicates that the quality of the graphite strip grown for 4 min is not uniform and continuous for $10 \mathrm{~min}$ and $7 \mathrm{~min}$.

Combined with the Raman spectrum analysis in 3.3.1, with the extension of growth time, the thickness of graphite strips on the copper surface increases, but the defect density also increases. The polarization curves in this section show that the corrosion resistance of graphene grown in $7 \mathrm{~min}$ is better than that of graphene grown in $10 \mathrm{~min}$ and $4 \mathrm{~min}$, that is, the quality of graphene grown in $7 \mathrm{~min}$ is the best, that is, the most uniform and continuous. The results show that the graphene with 10 min growth time has 3-4 layers, but its structure is disordered, and there are many defects such as grain boundary, which lead to the decline of corrosion resistance; while the graphene with 4 min growth time has about 2-3 layers, although the grain structure is the most orderly, but the local discontinuity of graphene film on copper surface leads to the decline of corrosion resistance.

The electrolyte of this test is $0.1 \mathrm{~mol} / \mathrm{L} \mathrm{NaCl}$ solution, because the corrosivity of $\mathrm{CL}$ is stronger than SO42 -, CO32 -, PO43 -, and the area of all working electrodes is LCM $\times$ LCM. The samples under each condition are randomly selected. The test results show that the average corrosion current of the samples with graphene film on the copper surface is one order of magnitude lower than that of pure copper, so it is not difficult to draw a conclusion that the graphite residue film prepared by atmospheric pressure vapor deposition method can effectively inhibit the corrosion of copper substrate.

\section{3 electrochemical impedance spectroscopy}

In this electrochemical impedance spectroscopy (EIS) test, the scanning frequency range is $0.01 \mathrm{~Hz} \sim 100000$ Hz. The impedance test data are fitted by zsimpwin software, and the curves of four groups of parallel samples are shown in Figure 6. 

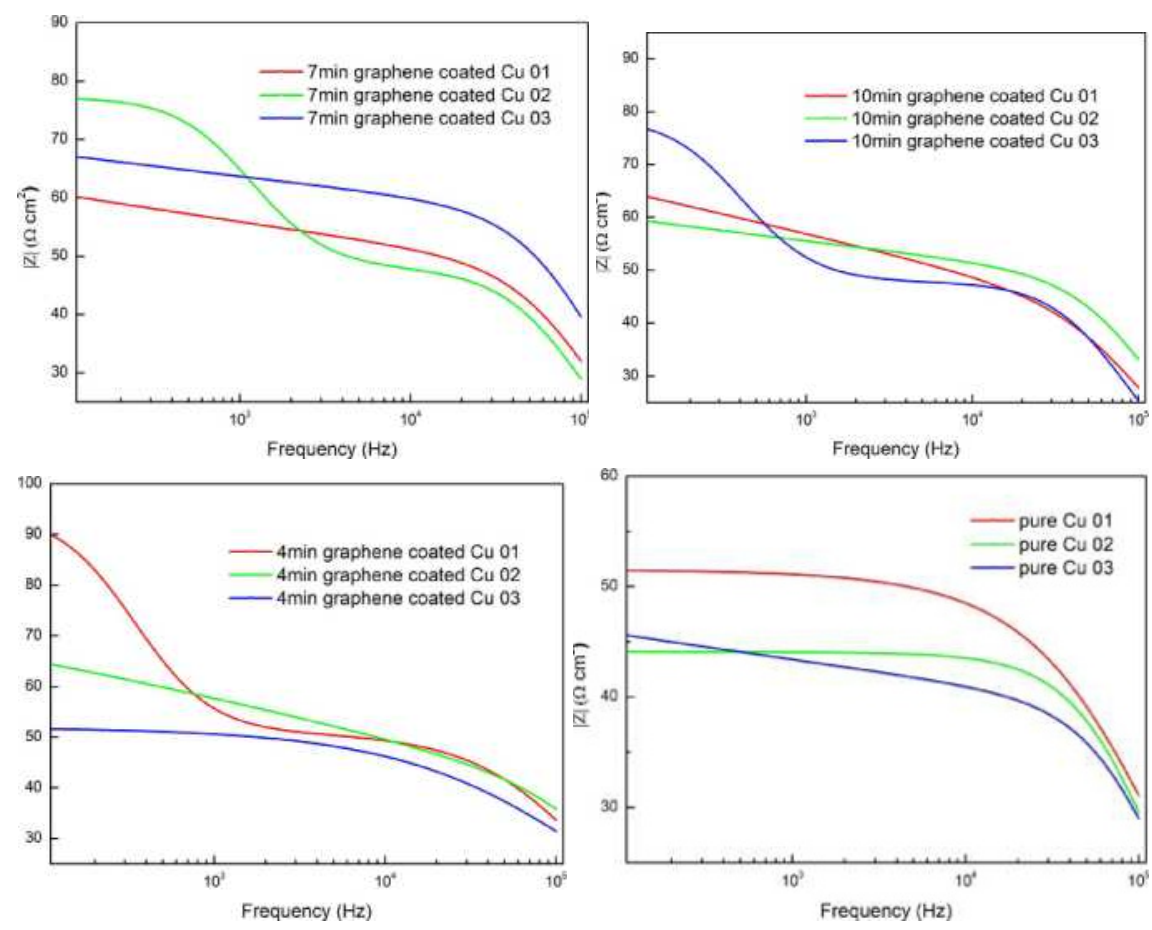

Fig 6 The Bode curve of parallel specimens

Fig. 6 is the baud diagram of pure copper and graphene coated copper samples with growth time of 4 min, $7 \mathrm{~min}$ and $10 \mathrm{~min}$ respectively. The relationship between frequency and modulus $/ \mathrm{Z} /$ is reflected, where $/ \mathrm{Z} /=(\mathrm{z} 2$ real $+\mathrm{z} 2$ imaginary $) 1 / 2$, zreal is the real part of impedance, and the imaginary part of impedance. The modulus / Z / at low frequency reflects the corrosion resistance of the material. Three parallel samples are set for each group of samples. It can be seen from the figure that the curves of parallel samples are relatively close. Take the middle value for final comparison, as shown in

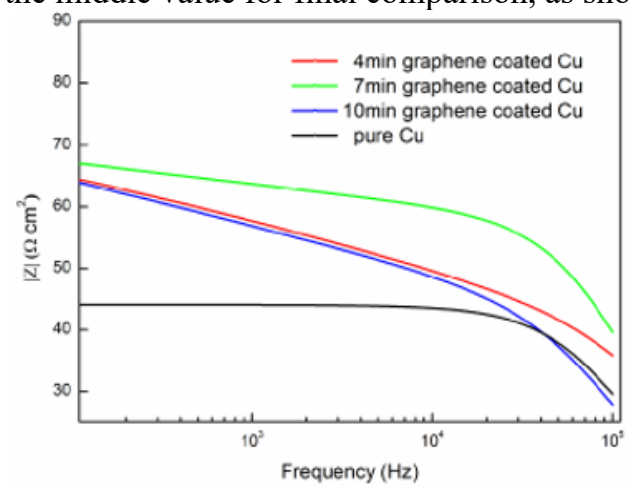

Fig 7 The final contrast chart of Bode curve

It can be seen from Figure 7 that at low frequency, the modulus / Z / of copper samples with graphene on the surface is higher than that of pure copper. The modulus / Z / of copper samples with growth time of 7 min is the largest at low frequency, which is about 1.5 times of that of pure copper. The modulus / Z / of copper samples with growth time of $4 \mathrm{~min}$ and $10 \mathrm{~min}$ is slightly smaller, which indicates that the corrosion resistance of copper samples with growth time of $7 \mathrm{~min}$ is the best, and that of copper samples with growth time of $4 \mathrm{~min}$ and $10 \mathrm{~min}$ is the best The results show that the graphene film prepared by atmospheric pressure vapor deposition can effectively inhibit the corrosion of copper substrate, and the best growth time is $7 \mathrm{~min}$.

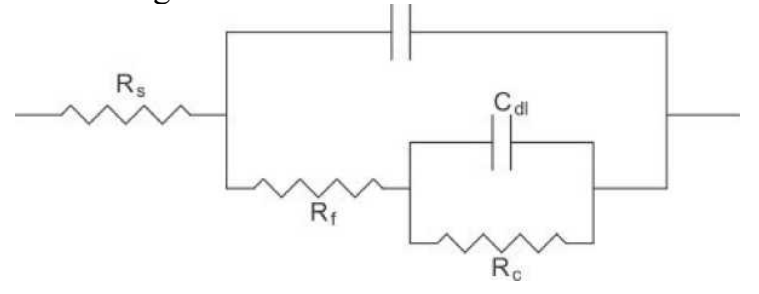

Fig 8 The electrical equivalent circuits of the graphene coated copper

In order to quantify the electrochemical corrosion phenomenon in this study and better analyze the corrosion mechanism, the electrical equivalent circuits are given, as shown in Figure 8. The relationship between copper matrix / graphite coating / electrolyte and copper matrix / copper oxide or hydroxide / electrolyte was studied for the samples with dilute graphite coating.

QF: surface coating corner element CPE (constant phase element), which is affected by the electrode surface activity, roughness, electrode pore, current and potential distribution and other factors, it represents the conductive path of the electrode surface; CDL: capacitance between metal and electrolyte, which is directly related to the area of metal substrate exposed in the electrolyte;

Re: interface resistance between metal and electrolyte;

$\mathrm{RF}$ : pore resistance;

Rs: resistance of electrolyte.

Re: interface resistance between metal and electrolyte;

$\mathrm{RF}$ : pore resistance;

Rs: resistance of electrolyte. 
Table2 Calculated parameters of the EEC for the specimens

\begin{tabular}{|c|c|c|c|c|}
\hline & Pure $\mathrm{Cu}$ & $\begin{array}{c}4 \text { min graphene } \\
\text { coated } \mathrm{Cu}\end{array}$ & $\begin{array}{c}7 \text { min graphene } \\
\text { coated } \mathrm{Cu}\end{array}$ & $\begin{array}{c}\text { 10min graphene } \\
\text { coated } \mathrm{Cu}\end{array}$ \\
\hline $\mathrm{Rf}(\Omega \mathrm{cm} 2)$ & 44.08 & $2.15 \times 10^{-5}$ & $1.0 \times 10^{-2}$ & $2.75 \times 10^{-6}$ \\
\hline $\mathrm{Rc}(\Omega \mathrm{cm} 2)$ & 16.89 & 89.11 & 1050 & 107.6 \\
\hline $\mathrm{Rf}+\mathrm{Rc}(\Omega \mathrm{cm} 2)$ & 61 & 89 & 1050 & 108 \\
\hline $\mathrm{Qf}(\mathrm{F} / \mathrm{cm} 2)$ & $6.12 \times 10^{-8}$ & $1.57 \times 10^{-3}$ & $1.28 \times 10^{-2}$ & $2.95 \times 10^{-3}$ \\
\hline $\mathrm{n}$ & 0.96 & 0.16 & 0.023 & 0.12 \\
\hline $\mathrm{Cdl}(\mathrm{F} / \mathrm{cm} 2)$ & $3.87 \times 10^{-2}$ & $1.85 \times 10^{-8}$ & $2.8 \times 10^{-8}$ & $3.9 \times 10^{-8}$ \\
\hline Chi squared value & $6.2 \times 10^{-3}$ & $3.1 \times 10^{-3}$ & $2.3 \times 10^{-3}$ & $7.1 \times 10^{-3}$ \\
\hline
\end{tabular}

Table 2 shows the values of the parameters of the equivalent circuit processed by zsimpwin software. The values of graphene coated copper are larger than that of pure copper, and the samples with graphene growth time of $7 \mathrm{~min}$ are the largest, which indicates that graphene film can well separate the copper matrix from the electrolyte and significantly increase the resistance between their interfaces. The arithmetic sum of $\mathrm{RC}$ and $\mathrm{RF}$ represents the corrosion resistance of the material, i.e. rpurccu $=61 \mathrm{dcm} 2$. The resistance of samples with graphene growth time of $4 \mathrm{~min}, 7 \mathrm{~min}$ and $10 \mathrm{~min}$ is 1.5 times, 17 times and 1.8 times of that of pure copper respectively, indicating that the sample with graphene growth time of 7 Min has the strongest corrosion resistance. Compared with the value of CDL, the capacitance of the substrate and coating of the sample with graphene on the surface is smaller than that of pure copper, which indicates that the graphene covers the copper substrate well, and the area exposed to the electrolyte is very small. The growth time of the sample is $4 \mathrm{~min}, 7 \mathrm{~min}$ and $10 \mathrm{~min}$ The CDL value decreases in turn, which indicates that the area and thickness of graphene on the copper surface increase with the extension of time, but the resistance of $7 \mathrm{~min}$ is the largest, which verifies the conclusion in 3.5.1 again, that is, the increase of graphene thickness and defect density also increase, so the corrosion resistance of graphene with growth time of $10 \mathrm{~min}$ is worse than that of $7 \mathrm{~min}, 4$ The results show that the corrosion resistance of graphene coating is the best under the condition of $7 \mathrm{~min}$ because of the discontinuity of thin graphite.

\section{Conclusion}

The polarization curve and electrochemical impedance spectrum show that the corrosion resistance of graphene grown for $7 \mathrm{~min}$ is the best. The polarization curve shows that the corrosion potential of graphene is about $0.4 \mathrm{~V}$ higher than that of pure copper, and the corrosion current density is about 1 order of magnitude lower than that of pure copper. The electrochemical impedance spectrum shows that the resistance of graphene is 1050 $\mathrm{qcm} 2$, about 17 times higher than that of pure copper.

The graphene with $7 \mathrm{~min}$ growth time has the best structure, quality and corrosion resistance. The graphite with growth time of $4 \mathrm{~min}$ has less residual layers, but the continuity is not good. The graphite with growth time of $10 \mathrm{~min}$ has more layers and higher defect density.

\section{Acknowledgement}

Science and technology project of China Southern Power Grid Co., Ltd., Project No.: G zkj xm20181985

\section{References}

1. De Heer W A, Berger C, Wu X S, et al, Epitaxial Graphene, Solid State Commun, 2007, 143: 92 100.

2. Kirkland N T Schiller T, Medhekar N, Exploring graphene as a corrosion protection barrier, Corros Sci, 2012, 56:1 4.

3. Prasai D, Tuberquia J C, Harl R R, et al, Graphene: corrosion-inhibiting coating, ACS Nano, 2012, 6(2)] $1 \sim 28$.

4. Dongwoo Kang, Jee Youn Kwon, Hyun Cho, et al, Oxidation Resistance of Iron and Copper Foils Coated with Reduced Graphene Oxide Multilayers, ACS Nano 2012,9: 7763 7769

5. Chi-Hao Chang, Tsao-Cheng Huang, Chih-Wei Peng, et al, Novel anticorrosion coatings prepared from polyaniline/graphene composites, Carbon, 2012, 50(14): 5044 5051.

6. Kotov N A, De'ka' ny I, Fendler J H, Ultrathin graphite oxide-polyelectrolyte composites prepared by self-assembly: transition between conductive and non-conductive states, Adv Mater, 1996, 8(8): 637641.

7. Stankovich S, Dikin D A, Piner R D, Synthesis of graphene-based nanosheets via chemical reduction of exfoliated graphite oxide, Carbon, 2007, 45 (7): $1558 \sim 1565$. 
8. Ferrari A C, Meyer J C, Scardaci V, et al? Raman spectrum of graphene and graphene layers, Phys Rev Lett, 2006, 97(18): 187-201.

9. Ni Z H, Wang H M, Kasim J, et al, Graphene thickness determination using reflection and contrast spectroscopy, Nano Online references will be linked to their original source, only if possible. To enable this linking extra care should be taken when preparing reference lists. 